\title{
Stabilized parametric Cooper-pair pumping in a linear array of coupled Josephson junctions
}

\author{
Mateusz Cholascinski and Ravindra W. Chhajlany \\ Institute of Physics, Adam Mickiewicz University, 61-614 Poznań, Poland
}

(Dated: October 1, 2018)

\begin{abstract}
We present an experimentally realizable stabilized charge pumping scheme in a linear array of Cooper-pair boxes. The system design intrinsically protects the pumping mechanism from severe errors, especially current reversal and spontaneous charge excitation. The quantum Zeno effect is implemented to further diminish pumping errors. The characteristics of this scheme are considered from the perspective of improving the current standard. Such an improvement bears relevence to the closure of the so-called measurement triangle (see D. Averin [Nature 434, 285 (2005)]).

PACS numbers: 74.81.Fa 03.65.Xp 73.23.-b 85.25.Cp
\end{abstract}

Adiabatic variation of parameters in a quantum system results in evolution in which population of each of the energy levels is conserved (adiabatic following). It is well known that, during such evolution, the energy levels acquire two types of phases: time dependent dynamical phases and time independent geometrical phases determined only by the geometry of the path traversed in parameter space. The property of adiabatic following can be also used to induce transport through a system, viz. a slowly transported potential well carries its bounded particles.

For closed paths, the initial and final states (usually) differ only by a phase factor [13], but the system evolution during the cyclic variation can still be characterized by non-vanishing transport properties. This phenomenon, known as parametric pumping, is observable in mezoscopic devices, where during each pumping cycle a definite charge is transported through the system [1, 2, 3, 4, [5, 6]. Quantum pumps are hoped to transform an AC signal of frequency $f$ into DC current given by the relation $I=f Q$, where $Q=n e$ for normal devices, and $Q=2 n e$ for superconducting pumps ( $n$ is the total charge transported per cycle). If operated with perfect accuracy, quantum pumps could be utilized to establish a standard of current. This is needed to close the measurement triangle relating voltage, current and frequency by fundamental constants [7]. However, nonadiabatic corrections, uncontrolled tunneling, and sensitivity of the devices to parametric fluctuations smear the output signal. An intristic stabilizing property, like quantization of magnetic flux, crucial for the voltage standard, is absent in the parametric charge pumps. Thus the only way to stabilize the performance is to optimize the design of such devices. E.g. in Ref. 5 enhanced control over the tunelling amplitudes in the so-called Cooper pair "sluice" resulted in improved characteristics of the pumping scheme.

In this Letter, we combine advantages of the controllable Josephson couplings used in the "sluice" and the array-like design of earlier proposals [4, 8]. Our scheme is analyzed in the framework of adiabatic passage in an effective two-level system. Electrostatic coupling of the Josephson junctions forming the arrays results in increased separation of the operational subspace from the excited states. The array design also suppresses the most severe process affecting the pumping accuracy - current reversal. This suppression increases exponentially with the number of junctions in the system. Since after each pumping sequence the system is to be found in a known charge state, we also make use of the quantum Zeno effect to diminish the population of unwanted states.

The core mechanism of the adiabatic passage can be understood using the simplest nontrivial - two-level system. Suppose that we start at degeneracy (zero field in the spin- $1 / 2$ language) and spin pointing "up" in the $z$ direction. Then the field is increased along the spin direction and traverses a path in the $x-z$ plane which approaches the degeneracy again from the negative $z$ direction. For adiabatic pulses, the system remains in the ground state and follows the field. However, at the end the spin points in the negative $z$ direction. This simple example shows that although the system is in a nondegenerate level during the passage and the traversed path in the parameter space is closed the resulting transformation is highly nontrivial as the spin direction is reversed. This is due to the level crossing occurring at the beginning and end of the path. For zero field the states are degenerate, and this freedom of defining the orthogonal basis results in discontinuity of the energy eigenstates.

A Cooper-pair box with tunable Josephson coupling 9] [see Fig. 1(a)], described by the Hamiltonian $H=-1 / 2 B_{z} \sigma_{z}-1 / 2 B_{x} \sigma_{x}$, where $B_{x} \equiv J(\Phi)=$ $2 J_{0} \cos \left(\pi \Phi / \Phi_{0}\right)$, and $B_{z}=4 E_{C}\left(1-2 n_{g}\right)$, mimics a spin$1 / 2$ system. Here $J_{0}$ is the Josephson coupling of the junctions in the SQUID (Superconducting Quantum Interference Device) coupling the box to the reservoir, $\Phi$ and $\Phi_{0}$ the external applied flux through the SQUID and flux quantum respectively, $E_{C}=e^{2} /\left(2 C_{g}+2 C_{J}\right)$ is the single-electron charging energy of the box and $n_{g}=C_{g} V_{g} / 2 e$ is the dimensionless gate charge. The twolevel approximation holds if the system is operated in the charge regime $\left(J_{0} \ll E_{C}\right)$ and $n_{g}$ is close to $1 / 2$. Then 

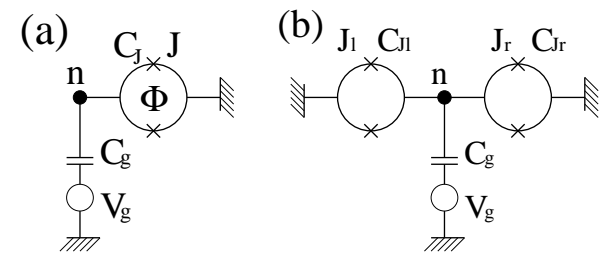

FIG. 1: (a) Cooper pair box [9]. Electrostatic potential of the superconducting island (black node) is controlled by the gate voltage $V_{g}$. The Josephson tunelling amplitude $J$ is tuned by external flux $\Phi$. (b) A Cooper-pair box with two SQUIDS.

the only relevant states are characterized by zero or one Cooper pair in the box, corresponding to spin parallel and antiparallel to the $z$ direction respectively. Performing the procedure described above on the system, we can thus transport a Cooper pair to or from a reservoir into an empty box, depending on the sequence of pulses.

To induce transport through the Josephson-junction system we use two SQUIDs which now serve as terminals [see Fig प(b)]. Using one of the terminals to transport a Cooper pair into the box (stage one) and another to transport the same pair to the reservoir (stage two) we generate current $I=2 e f$. Exemplary pulses of a full cycle are depicted in Fig. 2. No transport takes place during parts I and IV with the system in a definite (zero) charge state, so we can limit the cycle to the sequences II and III only. This simplification minimizes the cycle duration and eliminates degeneracies from the procedure (this system is identical to the Cooper pair "sluice" [5]).

Let us comment briefly on the possible sources of imperfections in the pump performance. The nonadiabatic corrections leave the system in an unknown superposition of charge states after the full cycles (instead of a definite charge state). These corrections whilst usually small, can accumulate over time. Fluctuations of external parameters affect the scheme similarly, though fluctuations in external flux have a much more severe effect than those in the gate voltage, especially when the SQUIDs are not ideally symmetric. The terminals cannot then be completely closed, due to residual Josephson coupling, and the fluctuations induce transitions between charge states. The error related to this feature is the hardest to eliminate - since there is nonzero tunneling to the reservoir via both terminals all the time. Cooper pairs can accidentally be transported in the wrong direction, leaving no trace of the error in cases when the the final charge state is identical to the expected state. In the following we show that the array design combined with Zeno projections strongly suppresses these pumping errors.

The system we consider is shown in Fig. 3(a). It is composed of $N$ Cooper-pair boxes. Nearest neighbours are coupled with dc-SQUIDs, leftmost and rightmost islands are coupled to the reservoirs via other SQUIDs (terminals). If the number of Cooper pairs on the is-

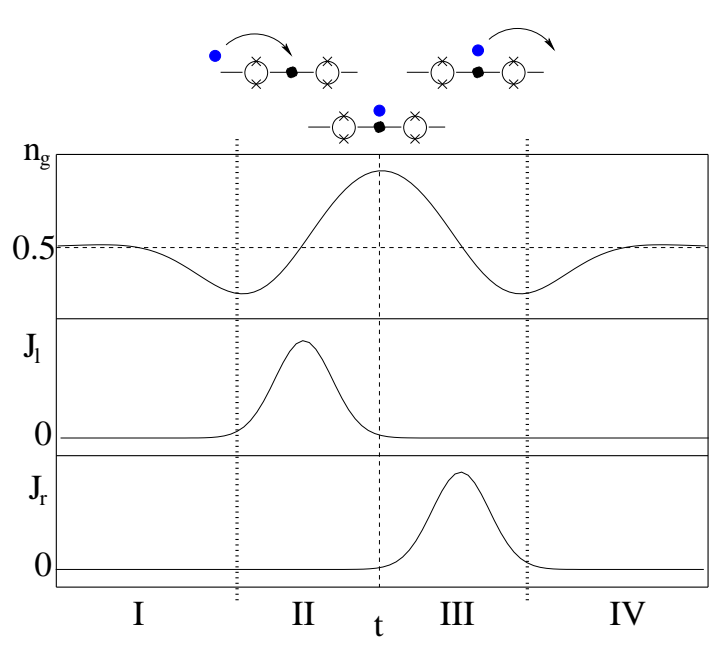

FIG. 2: A pulse sequence applied to the simple pump that induces flow of one Cooper pair through the system. The exact shape of, and area under the pulses do not influence the process as long as the adiabaticity condition holds.

lands is $\left\{n_{1}, n_{2}, \ldots, n_{N}\right\}$, the electrostatic energy of the system $E_{n_{1}, n_{2}, \ldots, n_{N}}=\sum_{i} E_{c(i)}\left(n_{i}-n_{g(i)}\right)^{2}+E_{m}^{i, i+1}\left(n_{i}-\right.$ $\left.n_{g(i)}\right)\left(n_{i+1}-n_{g(i+1)}\right)$. Here $E_{c(i)}$ are the charging energies of the islands and $E_{m}^{i, i+1}$ is the energy of electrostatic coupling between two neighboring islands, which is finite for finite capacitance of the coupling SQUID. If the dimensionless gate charges, $n_{g(i)}=C_{g(i)} V_{g(i)} / 2 e$ are close to $1 / 2$, the lowest energy states are characterized by either zero or one Cooper pair on each island. With this assumption we can again reduce the Hilbert space and map our system to a finite anisotropic Heisenberg spin$1 / 2$ chain in an external magnetic field. The Hamiltonian

$$
\begin{gathered}
H=-\frac{1}{2} B_{x}^{1} \sigma_{x}^{1}-\frac{1}{2} B_{x}^{N} \sigma_{x}^{N}-\frac{1}{2} \sum_{i=1}^{N} B_{z}^{i} \sigma_{z}^{i} \\
+\frac{1}{2} \sum_{i=1}^{N-1}\left[\Delta_{i, i+1} \sigma_{z}^{i} \sigma_{z}^{i+1}-J_{i, i+1}\left(\sigma_{+}^{i} \sigma_{-}^{i+1}+\sigma_{+}^{i+1} \sigma_{-}^{i}\right)\right]
\end{gathered}
$$

is characterized by constant electrostatic coupling amplitudes $\Delta_{i, i+1}$ and tunable parameters - $B_{x}^{1, N}$ - the Josephson coupling of the leftmost and rightmost SQUIDs, $B_{z}^{i}$ electrostatic potential of the islands and $J_{i, i+1}$ - Josephson coupling between neighbouring islands. In general the electrostatic coupling has a finite range determined by the screening length $\lambda=\sqrt{C_{J} / C_{g}}[10]$. As discussed below, we consider the regime in which the electrostatic coupling is much weaker than the on-site electrostatic energy. This implies that $\lambda \ll 1$ and we can thus limit ourselves to nearest neighbour electrostatic coupling only.

Because of the electrostatic interaction, for $n_{g}^{i}$ close to $1 / 2$ the most favorable charge states are antiferromagnetic $(|010101 \ldots\rangle$ and $|101010 \ldots\rangle)$. The pumping procedure is a simple generalization of the scheme described for one island and two terminals. We start with one of 


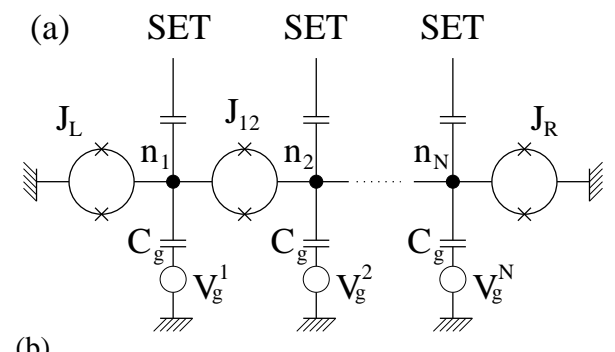

(b)

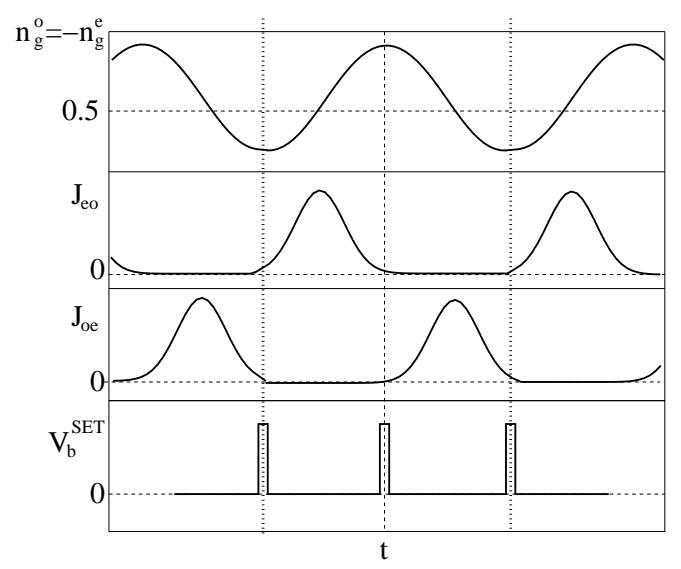

FIG. 3: (a) The stabilized Cooper-pair pump. It is operated similarly to the single-island system - identical pulse sequence is applied to every second junction (island) (b). Single electron transistors (SET) serve as the dephasing elements. They are biased with short voltage pulses every time the pump should be in a definite charge state [lowest plot in (b)].

the antiferromagnetic states and transfer the charge of every island to the right by two sites to achieve pumping. This can be implemented by applying identical pulse sequence to every second island (and SQUID), i.e. $B_{x}^{1}=$ $J_{2,3}=J_{4,5}=\ldots \equiv J_{e o}, J_{1,2}=J_{3,4}=\ldots=B_{x}^{N} \equiv J_{o e}$, $B_{z}^{1}=B_{z}^{3}=\ldots \equiv B_{z}^{o}$, and $B_{z}^{2}=B_{z}^{4}=\ldots \equiv B_{z}^{e}[$ see Fig. [3(b)] (o,e denote odd and even islands respectively). Here we focus on systems with odd number of islands then the number of coupling SQUIDs is even, and exactly half of them are active during each step of the procedure. This symmetry simplifies our analysis but is not crucial.

The procedure, even for the single-island realization, takes place in nondegenerate ground state. Due to the avoided degeneracy, spontaneous transitions to unwanted levels cost energy and are thus suppressed. The chain design additionally provides the following advantage: on the one hand an energy gap between the antiferromagnetic states and the remaining subspace $\Delta E \geq$ $\min \left\{\Delta_{i, i+1}\right\}$, while on the other hand accidental transitions between the antiferromagnetic states need simultaneous transport through $(N+1) / 2$ junctions - another effect which diminishes exponentially with $N$ (see Fig. 4(b) and explanation below). The most evident advantage of this approach is, however, elimination of transport in the wrong direction. Let us analyze the mechanism rul- ing this process. Suppose that we start with the state $\mid 101010 \ldots$. . . During the first part of the cycle we want to shift the charge configuration by one site to the right, i.e. to arrive at $|010101 \ldots\rangle$. As mentioned before, the residual Josephson coupling of closed SQUIDs leads to two ways of arriving at the same state. One could anticipate interference effects here, but since the residual coupling is a fluctuating quantity (controlled by fluctuating magnetic field) we treat the process as incoherent. A simple analysis is based on the assumption that a Cooper pair is transported through an open (correct) SQUID with probability $p$, and through closed (wrong) SQUID with the probability $q=1-p$. The measure of this pumping error for each island might be the ratio $q / p$. For a chain of $N$ junctions the probability of correct shift becomes $\propto p^{N / 2}$, and of incorrect shift $\propto q^{N / 2}$. Hence the pumping error $(q / p)^{N / 2}$ decreases exponentially with the number of junctions in the chain. More rigorous analysis requires calculation of the energy difference between the two lowest energy levels for $B_{z}^{i}=0$. Indeed, in this case the lowest energy eigenstates are symmetric and antisymmetric superpositions of the antiferromagnetic states (with some addition of other charge states), and the energy difference, being the rate of phase progression, in the charge basis is equivalent to the rate of transition. Fig. 4(a) shows the results for various values of the maximum Josephson coupling of the SQUIDs. $T$ is the rate calculated for the desired transitions only (assuming no residual coupling), $t$ is calculated for the wrong path. Then, the time of single operations should be much larger than $1 / T$ for the adiabatic condition to be satisfied. The numerical calculation is performed for a realistically achievable residual coupling equal to $1 \%$ of the maximum Josephson coupling of the SQUIDs. The values of the electrostatic coupling amplitudes, and maximum Josephson couplings are for simplicity assumed identical for each junction (this is, again, not crucial and our observations are valid also for different settings). The pumping error vs. $N$ is plotted in Fig. 4 (a). The dependence is clearly exponential, and very weakly depends on the value of the maximum Josephson coupling. However, the value of $T$ itself should not decrease too strongly with increasing $N$ as it determines the adiabatic rates. From Fig. 4 (b) we can see that the ratio $J_{\max } / \Delta$ should be as close to 1 as possible to avoid the effects of exponential suppression. For this case, $T / \Delta \approx 1$, where $\Delta$ can be chosen large thus yielding a very comfortable adiabatic condition. Note that even for $J_{\max } / \Delta \approx 1$ we can still work in the reduced Hilbert space, as long as the charging energies of individual islands are much larger than $J_{\max }$ (this means that the system should be operated in the charging regime with weak electrostatic coupling). The exponential suppression of the energy gap between the lowest states implies that unwanted transitions between the antiferromagnetic states also diminish very rapidly with increasing $N$ (note that this suppres- 

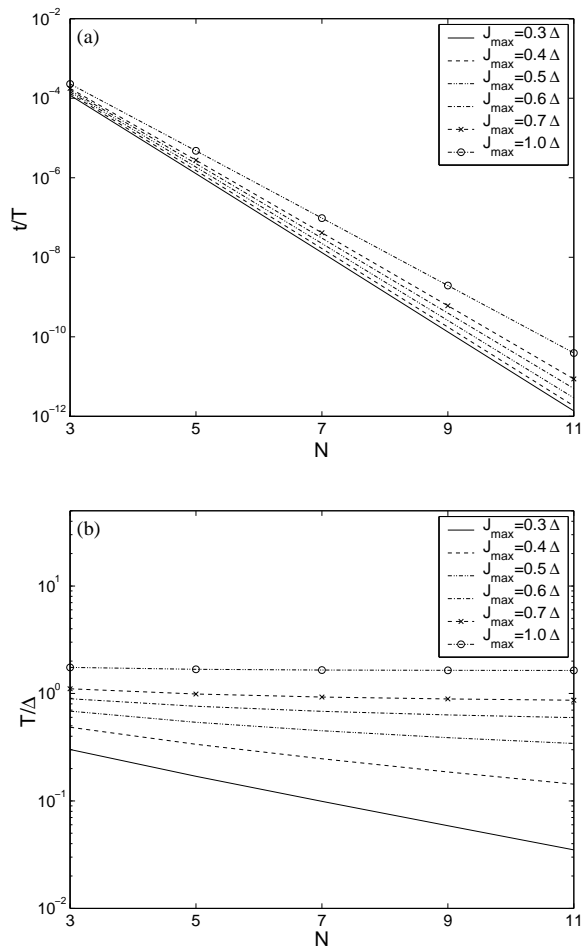

FIG. 4: (a) Pumping error $t / T$ as a function of $N$. For logarithmic vertical axis the dependence is linear, implying exponential suppression of the error with increasing $N$. (b) Transition rates between the charge states as a function of $N$.

sion for very small, erroneous, values of Josephson coupling is much stronger than for moderate values, expected for open terminals). This of course does not mean that the optimal setup should be represented by as long an array as possible. Most probably there is some moderate number of junctions for which the pumping errors are strongly suppressed, and the decoherence effects typical for macroscopic system are yet not severe. However, examination of the long-chain limit goes beyond our analysis and would be appropriate for a separate publication.

Although during the passage the state of the system is a superposition of numerous charge configurations, when all Josephson couplings are switched off (or rather intended to be) the pump should be in one of the antiferromagnetic states. All other components (nonadiabatic corrections, residual Josephson coupling effects) contribute to pumping errors. The optimal solution would be to project the state onto a charge state by performing charge measurement. Indeed, if the measured state is very close to an eigenstate of the measured observable, the measurement takes the state closer to the eigenstate. If frequently repeated, the measurement can diminish the probability of transition to other eigenstates arbitrarily. This phenomenon, known as quantum Zeno effect [11], can be utilized also in our system. The charge measurement can be performed by a single-electron tran- sistor (SET) coupled capacitively to the superconducting island 12] [see Fig. 3(a)]. This measurement is characterized by few time scales, the dephasing time $\tau_{\phi}$, during which the off-diagonal density matrix elements (in the basis of the measured observable) vanish, the measurement time, $\tau_{\text {meas }}$, after which the information about the charge can be read out, and the mixing time, after which the back action of the SET on the island destroys the information about the state. The parameters can be selected in such way that $\tau_{\text {mix }} \geq \tau_{\text {meas }} \geq \tau_{\phi}$. Since the actual information about the state is not important, and only the dephasing mechanism modifies the state, the quantum Zeno projection can be realized within a relatively short time. Moreover, even if the time available is too short for the off-diagonal entries of the density matrix to vanish, partial dephasing still brings the system closer to the desired charge state. The system can be thus dephased each time the system is supposed to be in a definite charge state by switching on the bias voltage of the SETs for a very short time interval.

In summary, the presented scheme provides an accurate pumping procedure. The array design exponentially suppresses the most severe, untracable error of reversed current flow and diminishes errors due to undesired charge configurations. These errors can be further minimized by repeated dephasing with single-electron transistors.

The authors thank J. Siewert, O. Buisson, and S. Paraoanu for discussions. M.C. acknowledges support from Polish Ministry Grant No. 1 P03B 06730.

[1] H. Pothier, P. Lafarge, C. Urbina, D. Esteve, and M.H. Devoret, Europhys. Lett. 17, 249 (1992).

[2] J.P. Pekola, A.B. Zorin, and M.A. Paalanen, Phys. Rev. B 50, 11255 (1994).

[3] M.W. Keller, J.M. Martinis, N.N. Zimmerman, A.H. Steinbach, Appl. Phys. Lett. 69, 1804 (1996).

[4] J. P. Pekola, J. J. Toppari, M. Aunola, M. T. Savolainen, and D. V. Averin, Phys. Rev. B 60, R9931 (1999).

[5] A. O. Niskanen, J. P. Pekola, and H. Seppä, Phys. Rev. Lett. 91, 177003 (2003).

[6] A. O. Niskanen, J. M. Kivioja, H. Seppä, and J. P. Pekola Phys. Rev. B 71, 012513 (2005)

[7] K. K. Likharev, and A. B. Zorin, J. Low Temp. Phys. 59, 347 (1985).

[8] J. J. Toppari, J. M. Kivioja, J. P. Pekola, M. T. Savolainen, J. Low Temp. Phys. 136, 57 - 91 (2004)

[9] Yu. Makhlin, G. Schön, and A. Shnirman, Nature 398, 305 (1999).

[10] R. Fazio, K-H. Wagenblast, C. Winkelholz, G. Schön, Phys. B 222, 364 (1996).

[11] B. Misra and E. C. G. Sudarshan, J. Math. Phys. 18, 756 (1977).

[12] Yu. Makhlin, G. Schön, and A. Shnirman, Rev. Mod. Phys. 73, 357 (2001)

[13] M. V. Berry, Proc. R. Soc. London, Ser. A 392, 45 (1984). 
